\title{
$O T X 2$ is a therapeutic target for retinoblastoma and may function as a common factor between C-MYC, CRX, and phosphorylated RB pathways
}

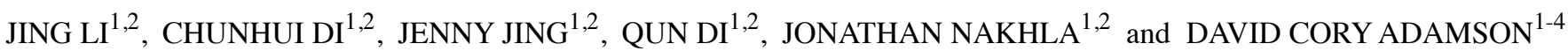 \\ ${ }^{1}$ Preston Robert Tisch Brain Tumor Center, Departments of ${ }^{2}$ Surgery (Neurosurgery) and ${ }^{3}$ Neurobiology, \\ Duke Medical Center; ${ }^{4}$ Neurosurgery Section, Durham VA Medical Center, Durham, NC, USA
}

Received November 10, 2014; Accepted December 19, 2014

DOI: $10.3892 /$ ijo.2015.3179

\begin{abstract}
The homeobox transcription factor orthodenticle homeobox 2 (OTX2) plays a critical role in very early neurogenesis, but can become oncogenic when aberrantly expressed later in life. We previously discovered its novel oncogenic role in the malignant childhood brain tumor medulloblastoma and hypothesize an oncogenic role in retinoblastoma. Primary retinoblastoma tumors and cell lines were analyzed by quantitative-PCR, immunoblotting and immunohistochemistry for OTX2. The effect of modulating OTX2 expression on tumorigenesis was tested pharmacologically and by siRNA. A lentiviral shRNA-engineered vector was used for conditional knockdown studies on tumor growth in vivo. A luciferase reporter assay was used to analyze ATRA's effect on OTX2's promoter. In this study on retinoblastoma, OTX2 was frequently amplified and/or overexpressed in primary tumors and cell lines. Knockdown of OTX2 expression by siRNA or pharmacologic inhibition by all-trans retinoic acid (ATRA) repressed OTX2 expression and cell proliferation and significantly decreased tumor growth in vivo. Loss of OTX2 expression also resulted in decreased expression of C-MYC and CRX, genes previously implicated in retinoblastoma tumorigenesis. Loss of OTX2 expression increased the phosphorylation of RB, a potential mechanism of modulating cell proliferation. Aberrant expression of OTX2 may contribute to the development of retinoblastoma. OTX2 may serve as a common transcription factor that interlinks multiple tumordriving pathways. These results also show that OTX2 can be genetically and pharmacologically targeted, providing an exciting new therapeutic option that may be less toxic and more efficacious than current treatments.
\end{abstract}

Correspondence to: Dr Cory Adamson, Preston Robert Tisch Brain Tumor Center, Duke Medical Center, DUMC Box 2624, Durham, NC 27712, USA

E-mail: cory.adamson@duke.edu

Key words: retinoblastoma, oncogene, OTX2, MYC, CRX, RB

\section{Introduction}

Retinoblastoma is the most common malignant intraocular tumor in children, typically occurring before 5 years of age $(1,2)$. Retinoblastoma affects $\sim 1$ in 18,000 children and can become fatal if left untreated $(1,3,4)$. The tumor is often initiated by the biallelic loss of the retinoblastoma tumor suppressor gene $(R B 1)$ and progresses quickly by accumulating additional genetic lesions and/or epigenetic changes in key cancer pathways (3). Surgery is typically the initial treatment followed by non-specific radiotherapy and/or chemotherapy. There are no current therapies that specifically target a unique molecular defect intrinsic to retinoblastoma. The non-specific radio- and chemotherapy treatments can have side effects including cognitive decline and secondary malignancy, which is especially worrisome in such a young susceptible patient population (5-7). Approximately $1 \%$ of patients with retinoblastoma in both eyes develop second non-ocular tumors each year and the cumulative probability of death from these secondary malignancies is $26 \%$ at 40 years after diagnosis $(8,9)$. In order to identify prognostic factors and develop more efficacious and safer treatments, a better understanding of the complex genomic and proteomic regulatory pathways that control the development of retinoblastoma is needed. Targeted chemotherapeutical agents personalized to specific tumor biology have the tremendous potential to be more clinically effective with less toxicity.

We previously identified the oncogene orthodenticle homeobox 2 (OTX2) in the malignant childhood brain tumor medulloblastoma $(10,11)$. OTX2 is a member of the highly conserved family containing the bicoid-like homeodomain transcription factors that control the developmental programs underlying brain morphogenesis and plays a role in tumorigenesis if aberrantly expressed later in life (12). This gene is expressed in the metencephalon (cerebellum precursor), diencephalon (thalamus and pineal gland precursor) and developing eye. OTX2 is also expressed in the developing retinal pigment epithelium (RPE) of the orbit (13). OTX2 is clearly important to the development, maturation and function of retinal cells. It is expressed in retinal photoreceptor and bipolar precursor cells $(14,15)$. The cell of origin for retinoblastoma is speculated by some to reside in the RPE. Serial Analysis of Gene 
Expression (SAGE) shows that the retina is one of the very few normal adult tissues that continue to express the gene, albeit at very low levels (http://cgap.nci.nih.gov/SAGE).

Our recent evidence has convincingly shown that OTX2's aberrant overexpression results in tumorigenesis, notably as medulloblastoma located in the cerebellum $(10,11)$. With genome-wide expression analysis, we identified genomic amplification and overexpression of OTX2 in medulloblastoma (10). A positive correlation of OTX2 overexpression with aggressive tumor behavior (e.g., anaplasia) and worse patient survival was also evident (11). To demonstrate its oncogenic ability, we showed that overexpression of OTX2 in non-tumorigenic cells produced tumors (11). Additionally, OTX2 regulates the medulloblastoma oncogene MYC (11). These results suggest that OTX2 plays a role in initiation and maintenance of a large subset of tumors and thus represents a promising specific therapeutic target.

Interestingly, three different malignant tumors (pineoblastoma, retinoblastoma and medulloblastoma) can occur in the same individual (16-18). The well-known syndrome of trilateral retinoblastoma includes inherited unilateral or bilateral retinoblastoma along with pineoblastoma, but reports of concurrent medulloblastoma have also been seen (16-18). Interestingly, OTX2 controls the cell fate of photoreceptor and pineal gland cells (14), suggesting a potential common cause of tumors in these tissues. Because of the ontogenetic relationship of the common expression of OTX2 in the developing precursor tissues to these adult structures, the strikingly common histological appearance of these tumors and common genetic aberrations (16,19-21), we hypothesize that OTX2 may serve a similar oncogenic role in retinoblastoma.

\section{Materials and methods}

Tissue samples. Retinoblastoma cell lines Y79 and WERI were purchased from American Type Culture Collection (ATCC) (Manassas, VA, USA). Medulloblastoma cells lines D425MED, D581MED and D324MED were obtained from the Duke Preston Robert Tisch Brain Tumor Center Biorepository. Acquisition of tissue specimens was approved by the Duke University IRB (\#Pro00008208) and done in accordance with HIPPA regulations.

Cell culture. D425MED was selected as an OTX2-positive control and D581MED/D324MED were selected as OTX2negative controls based on our prior data (10). Retinoblastoma cell lines were cultured using RPMI-1640 medium with 10\% FBS (Life Technologies; Grand Island, NY, USA), while medulloblastoma cell lines were cultured using a stock solution of $1 \mathrm{X}$ zinc option, HEPES and sodium bicarbonate (Life Technologies). Cells were maintained at $5 \% \mathrm{CO}_{2}$ and $37^{\circ} \mathrm{C}$.

Serial analysis of gene expression data analysis. The serial analysis of gene expression (SAGE) data were obtained from the National Center for Biotechnology Information Cancer Genome Anatomy Project repository (http://cgap.nci.nih.gov/ SAGE). The presence of OTX2 SAGE tags in a total of 157 tumors and 54 normal human tissues was identified by using the SAGE Anatomic Viewer.
Quantitative real-time PCR. Q-PCR (Bio-Rad, Hercules, CA, USA) was used to determine $O T X 2$ copy number changes between normal human DNA and retinoblastoma primary tumors or cell lines. Genomic DNA was isolated by DNeasy Blood \& Tissue kit (\#69504; Qiagen, Valencia, CA, USA) from 6 primary tumors (Rb1-6), cell lines Y79 and WERI, D425MED (positive control) and D581MED (negative control). Line 1 was used to normalize DNA content.

Immunohistochemistry. Immunohistochemistry (IHC) was used to evaluate protein expression and relationship with cellular morphology on primary and mouse xenograft tumors. For IHC, 5- $\mu \mathrm{m}$ paraffin-embedded tumors were obtained, deparaffinized by xylene and hydrated with a series of graded alcohols. The sections were then washed with distilled water and incubated in citrate buffer. Hydrogen peroxide $(0.3 \%)$ was used to block endogenous peroxidase activity. Sections were blocked with $1 \%$ bovine serum albumin (BSA) and $20 \%$ mouse serum (\#M5905, Sigma-Aldrich, St. Louis, MO, USA) in PBS. The sections were then screened with anti-OTX2 antibody at 1:200 (\#MAB1979, R\&D Systems, Minneapolis, MN, USA) overnight at $4^{\circ} \mathrm{C}$, followed by HRP-conjugated anti-goat secondary antibody at 1:200 (\#A8919, Sigma-Aldrich) for $1 \mathrm{~h}$ at room temperature.

Immunoblotting. Immunoblotting was used to detect the expression levels of OTX2, MYC, CRX and phosphorylated $\mathrm{RB}$ (pRB). Per standard protocol, $50 \mu \mathrm{g}$ cell protein isolates were prepared, denatured by heating at $97^{\circ} \mathrm{C}$ for $7 \mathrm{~min}$, loaded on to polyacrylamide gels, electrophoresed at $180 \mathrm{~V} 45 \mathrm{~min}$ and transferred to a nitrocellulose membrane at $15 \mathrm{~V}$ for $30 \mathrm{~min}$ (Bio-Rad). Membranes were blocked with 5\% non-fat milk and probed using primary antibodies (anti-OTX2, 1:5,000, MAB1979, R\&D Systems; anti-GAPDH, 1:3,000, \#sc-365062, Santa Cruz Biotechnology, Santa Cruz, CA, USA; anti-MYC, 1:1,000, \#9402, Cell Signaling Technology, Danvers, MA, USA; anti-CRX, 1:1,000, \#C7498, Sigma-Aldrich; anti-pRB, 1:1,000, \#9308, Cell Signaling Technology) overnight at $4^{\circ} \mathrm{C}$ with gentle shaking. Subsequently, membranes were washed and probed with the appropriate HRP-conjugated secondary antibodies (OTX2 needed anti-goat, 1:5,000, Invitrogen, Carlsbad, CA, USA; GAPDH/MYC/CRX/pRB needed antirabbit, 1:3,000, Santa Cruz Biotechnology) for $1 \mathrm{~h}$ at room temperature with gentle shaking. Proteins were visualized with SuperSignal West Femto Chemiluminescent Substrate (\#34095, Thermo Scientific, Waltham, MA, USA) by autoradiography.

siRNA inhibition of OTX2. Y79 and WERI cell lines were either transfected with one of the two OTX2-specific siRNAs or with a non-specific scrambled siRNA control (siRNA\#1, GGAGGU GGCACUGAAAAUCtt; siRNA\#2, GGACACUA AUUCA UCUGUAtt; Ambion, Austin, TX, USA; MISSION siRNA Universal Negative Control, Invitrogen). Transfection was conducted with $100 \mathrm{nM}$ of each siRNA and Lipofectamine 2000 (siRNA to Lipofectamine 2000 ratio of 1:2; \#11668-019, Invitrogen). The cells were incubated with the siRNA at $37^{\circ} \mathrm{C}$ and harvested at 24, 48, 72 and $96 \mathrm{~h}$ to ascertain maximum knockdown by immunoblotting. Seventy-two hours was chosen as the best time-point by immunoblotting, so cells were 
harvested at that time for assays. For each siRNA, studies were done in triplicate.

Cell proliferation, apoptosis and colony formation assays. After confirming OTX2 inhibition by immunoblotting, functional in vitro assays were performed. Cell viability and proliferation was measured using the MTT Cell Proliferation assay (\#30-1010k, ATCC). After OTX2 inhibition, cells were allowed to grow for 7 days and MTT assay was performed (measured at OD540). Cell density was normalized to untreated cells. Cell apoptosis in response to OTX2 inhibition was measured using the FITC Annexin V Apoptosis Detection kit (\#556547, BD Pharmingen, San Diego, CA, USA), normalized to untreated cells and analyzed by flow cytometry. For colony formation, treated cells were plated at $5 \times 10^{3}$ in triplicates in $0.5 \%$ agarose-coated 24 -well plates and the number of colonies was counted at 2 weeks.

Pharmacologic inhibition of OTX2. All-trans-retinoic acid (\#302-79-4, ATRA, Sigma-Aldrich) in DMSO (\#D8418, SigmaAldrich) at various concentrations $(0,0.5,2,5$ and $10 \mu \mathrm{M})$ was added to the cells. Time experiments were done to ascertain maximum reduction in OTX2 expression by immunoblotting. Maximum reduction in OTX2 expression was obtained after $72 \mathrm{~h}$. Similar functional assays were done in triplicate.

Conditional knockdown of OTX2 for in vivo studies. Using the open reading frame of OTX2 from GenBank (http://www. ncbi.nlm.nih.gov/genbank/), we designed 2 OTX2-specific and 1 non-specific scrambled shRNA with the Invitrogen BLOCK-iT RNAi Designer (http://rnaidesigner.invitrogen. com/rnaiexpress/). The sequence for shRNA\#1 was CGCG TCCCCGCTTGGATTATAAAGATCATTCAAGAGATGAT CTTTATAATCCAAGCTTTTTGGAAAT; shRNA\#2 was CGCGTCCCCGAGCTGCACTGAAACTTTATTCAAGAG ATAAAGTTTCAGTGCAGCTCTTTTTGGAAAT; scrambled shRNA was CGCGTCCCCGTATCGGATAATATCAGTAT TCAAGAGATACTGATATTATCCGATACTTTTTGGAAAT (IDT, Coralville, IA, USA). The restriction enzymes $M l u \mathrm{I}$ and ClaI (\#R0198L and \#R0197L, New England Biolabs, Ipswich, MA, USA) were used to ligate the gene into pLVTHM (\#12247, Addgene, Cambridge, MA, USA) cloning vector. $M s c$ I and FspI (\#R0534M and \#R0135L, New England Biolabs) were used to ligate it into the lentiviral construct pLVCT-tTR-KRAB (\#11643, Addgene), which contained a green fluorescent protein (GFP) tag and a Tet-on system so that inserts are transcribed in the presence of doxycycline. HEK293 cells (\#CRL-1573, ATCC) were transfected with the lentiviral vector, the packing plasmid psPAX2 (\#12260) and the envelope plasmid pMD2.G (\#12259, Addgene) to make viral particles. Lentiviral particles were collected and concentrated (Amicon Ultra centrifuge filter, Millipore, Billerica, MA, USA). Y79 and WERI were infected with lentiviral particles of each shRNA ( $2 \mu \mathrm{l}$ of the virus per $2 \times 10^{5}$ cells). After $24 \mathrm{~h}$ of incubation, the cell media was changed and cells were allowed to grow for 5 days. Fluorescent microscopy confirmed GFP expression.

OTX2 promoter assay. HEK293 cells were plated in 24-well plates with $4 \times 10^{5}$ cells per well the day before transfection. These cells were confirmed not to have OTX2 expression by immunoblotting. The next day, the medium was changed by adding different doses of ATRA from 0, 0.5, 2, 5 and $10 \mu \mathrm{M}$ in triplicates. Plasmids pGL3-enhance-OTX2 and pRL-CMV were co-transfected into HEK293 cells by Lipofectamine 2,000 per protocol. Forty-eight hours later, cells were harvested and analyzed by dual luciferase reporter assay system (\#E1910, Promega, Madison, WI, USA).

In vivo tumor growth. Once a conditional knockdown system with shRNA was successfully established and confirmed via immunoblotting, the infected cells were harvested, washed by phosphate buffer saline and injected into mice to create a xenograft model for in vivo tumor growth studies. Immunodeficient athymic $n u / n u$ mice (Duke Cancer Center Isolation Facility; $\mathrm{n}=5$ per group) were injected with $1 \times 10^{7}$ cells on their flank subcutaneously with a 27-gauge needle per approved animal protocol (\#A054-10-03). Doxycycline (\#D9891, Sigma-Aldrich) was given orally to half the mice in their water ( $2 \mathrm{mg}$ doxycycline per $1 \mathrm{ml}$ water). Control groups included cells not infected with viral vectors, cells not infected with viral vector and given doxycycline, cells infected with viral vector containing scrambled shRNA and cells infected with viral vector containing scrambled shRNA and given doxycycline. Experimental groups included cells infected with viral vectors containing one of the two shRNAs and cells infected with viral vectors containing one of the two shRNAs and given doxycycline. The doxycycline water was changed twice per week. Tumor growth was measured in two dimensions and the study ended per approved animal protocol.

Statistical analysis. Experiments were done in triplicate. The significance level for all tests was set a priori at 0.05 . To compare the treatment and control means, Student's paired t-tests (2 tailed) were performed.

\section{Results}

OTX2 has high expression in retinoblastoma, minimal expression in normal retina and no expression in other normal tissues. In order to examine OTX2 expression, we evaluated OTX2 transcript levels by SAGE data mining of 10 libraries obtained from normal adult human tissues and tumors (http:// cgap.nci.nih.gov/SAGE). The data revealed an average tag density of 22 for retinoblastoma, compared to an average tag density of 5.7 for normal retina. Consistent with SAGE analysis, quantification of OTX2 mRNA by Q-PCR showed minimal expression in adult retina, no detectable expression in other normal adult tissues tested and no expression in 18 adult glioblastoma multiforme tumors (data not shown) (10).

OTX2 is overexpressed in primary retinoblastoma tumors and cell lines. OTX2 overexpression was seen in $86 \%(19 / 22)$ of primary adult retinoblastoma tumors tested (Fig. 1A). High expression (Fig. 1B, representative tumor with $>2$ intensity and $>50 \%$ of cells immunopositive) was seen diffusely throughout the entire tumors and did not correlate with any specific histological features including rosettes, vascularity, or necrosis. OTX2 was robustly overexpressed in Y79 and WERI (RB cell lines) (Fig. 1C). D425 and D324 medulloblastoma cells served as previously reported positive and negative controls, 


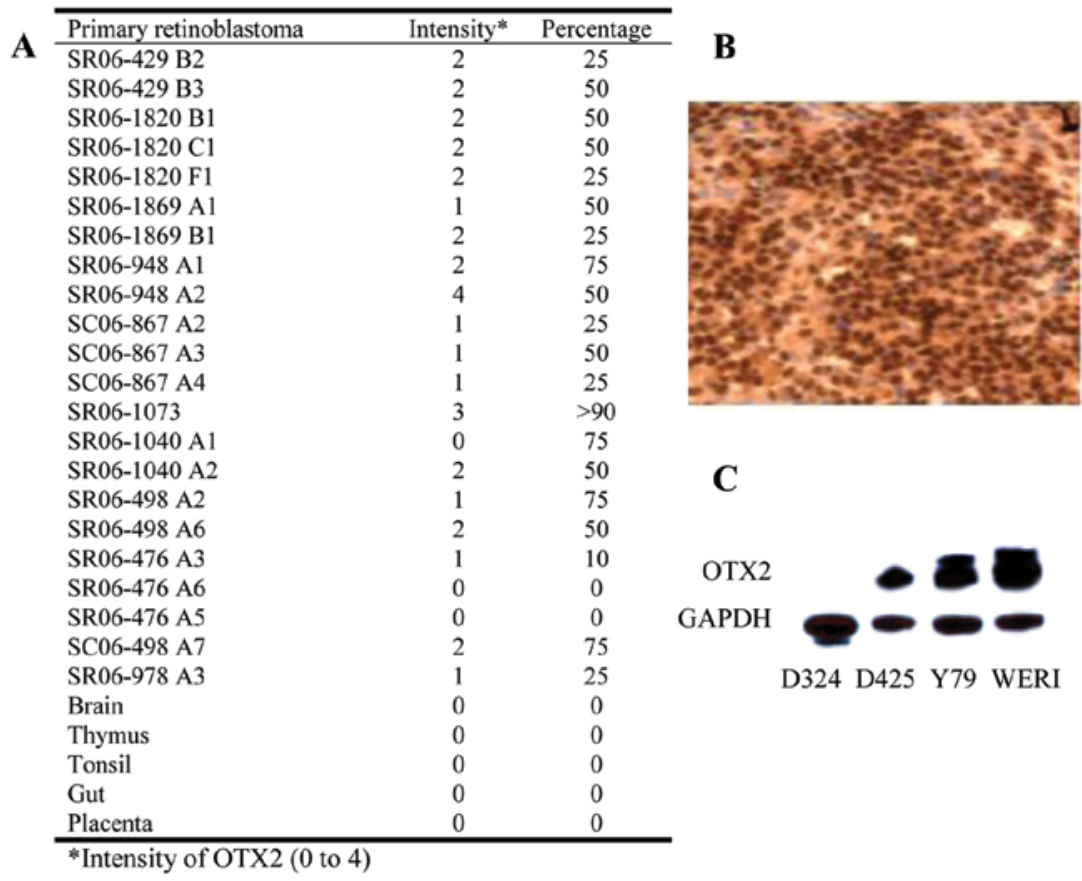

Figure 1. OTX2 is overexpressed in primary retinoblastoma tumors and cell lines. (A) Out of the 22 samples of primary retinoblastoma tumors examined, 86\% (19/22) showed high OTX2 expression. Normal tissues were negative. (B) Representative immunohistochemistry showing high OTX2-expressing primary retinoblastoma tumor (x40). Representative immunoblotting for OTX2 and GAPDH shows that OTX2 is robustly overexpressed in Y79 and WERI. GAPDH served as a housekeeping protein to demonstrate equal protein amounts. D425MED (positive control) shows the expected OTX2 expression whereas D324MED, an OTX2-non-expressing medulloblastoma cell line, shows no expression (negative control).
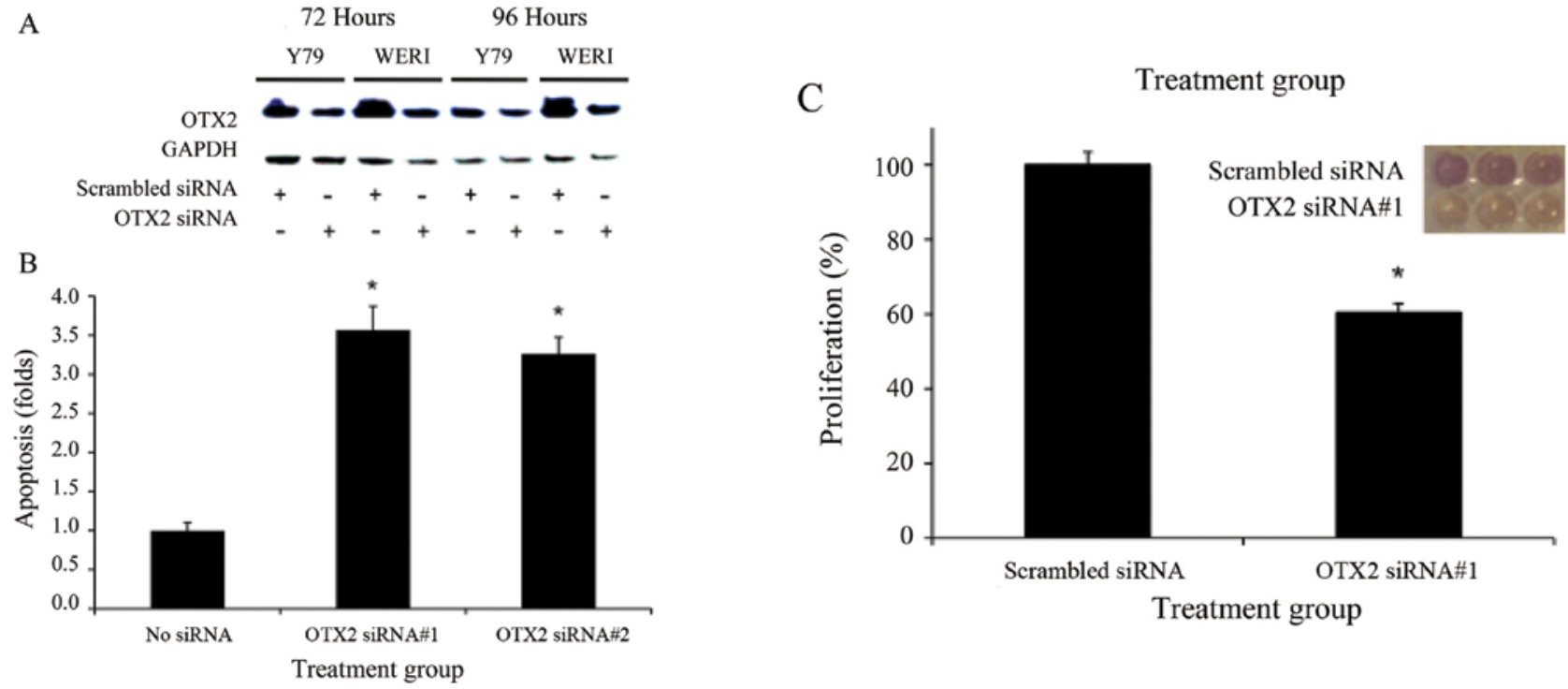

Figure 2. Inhibition of $O T X 2$ via siRNAs causes increased apoptosis and decreased proliferation in retinoblastoma cell lines. (A) Representative immunoblotting for OTX2 and GAPDH shows maximum OTX2 knockdown after $72 \mathrm{~h}$ of siRNA exposure for both Y79 and WERI. GAPDH served as housekeeping protein to demonstrate equal protein amounts. Densitometric analysis confirmed significant reduction in OTX2 with both siRNAs tested (p<0.05). (B) OTX2specific siRNA induces retinoblastoma cell apoptosis. Y79 and WERI were treated with the indicated OTX2-specific siRNAs or scrambled siRNA for $72 \mathrm{~h}$ and apoptosis was measured. (C) OTX2 gene knockdown by siRNA decreases cell proliferation. Y79 and WERI was treated with the indicated siRNAs for $72 \mathrm{~h}$ and allowed to grow for 7 days. Cell proliferation was then measured with the MTT assay. Asterisks (*) indicate significant differences when comparing each siRNA to the control ( $" \mathrm{p}<0.05)$.

respectively. Using Q-PCR, OTX2 amplification was observed in $33 \%(2 / 6)$ of the primary tumors tested (data not shown).

Inhibition of OTX2 causes increased apoptosis and decreased proliferation in retinoblastoma cell lines. After 72 and $96 \mathrm{~h}$ of exposure to siRNA specific for OTX2, there is a significant reduction of OTX2 expression in Y79 and WERI as normalized to scrambled siRNA treated cells (Fig. $2 \mathrm{~A},{ }^{*} \mathrm{p}<0.05$ ). Densitometric analysis confirmed significant reduction in OTX2 with both siRNAs tested ( $<<0.05)$. Inhibition of OTX2 
expression significantly increased the level of apoptosis in Y79 and WERI as compared to no inhibition by scrambled siRNA (Fig. 2B, " p<0.05). When measuring Annexin V levels, we routinely get $2-3 \%$ (or $<0.1$-fold change) apoptosis in our baseline, untreated cells. We also observed a significant reduction in cell proliferation after 7 days in cells treated with OTX2-specific siRNA, whereas minimal effect were seen using the scrambled-siRNA (Fig. $2 \mathrm{C},{ }^{*} \mathrm{p}<0.05$ ), normalized to untreated cells. Inhibition by two different siRNAs strongly supports specificity on the OTX2 gene. Furthermore, a dosedependent response to siRNA knockdown was demonstrated while selecting the optimal siRNA conditions.

Pharmacologic inhibition of OTX2 causes increased apoptosis, decreased proliferation and colony formation in retinoblastoma cell lines. We previously demonstrated that ATRA can pharmacologically inhibit OTX2 in medulloblastoma cells (10). We confirmed that OTX2 is repressed after ATRA treatment in retinoblastoma cells in a dose-dependent manner by immunoblotting (Fig. 3A). Densitometric analysis confirmed significant reduction (not shown). Inhibition of OTX2 expression with $2 \mu \mathrm{M}$ ATRA significantly increased cell apoptosis in Y79 (Fig. 3B, $\left.{ }^{*} \mathrm{p}<0.05\right)$. ATRA $(5 \mu \mathrm{M})$ increased cell death further at which dose this effect appeared to plateau since additional ATRA had similar effect. ATRA (2 $\mu \mathrm{M})$ also significantly reduced cell proliferation in Y79 (Fig. 3C, *p<0.05). Similarly, $2 \mu \mathrm{M}$ ATRA significantly reduced colony formation in Y79 (Fig. 3D, * p<0.05). Again, $5 \mu \mathrm{M}$ had additional effect on colony formation at which dose this effect plateaued. ATRA can target multiple molecular pathways, so we tested the effect of ATRA in cells transfected with a luciferase-driven OTX2 promoter. These cells did not have endogenous OTX2 expression. Fig. 3E shows that ATRA can specifically inhibit the OTX2 promoter in a dosedependent manner, suggesting a direct effect of this agent on OTX2 expression ( $\left.{ }^{*} \mathrm{p}<0.05\right)$.

In vivo inhibition of OTX2 in retinoblastoma xenografts reduces tumor growth and tumor size. To examine the oncogenic effects of OTX2 in vivo, we used a conditional knockdown strategy in xenograft flank models. We observed significant decreases in tumor growth when OTX2 was suppressed, when compared to controls (Fig. 4A, $\left.{ }^{*} \mathrm{p}<0.05\right)$. Tumors in the control group appeared significantly earlier ( 64 days; Fig. 4A, gray line) than the tumors in the knockdown group ( 152 days; Fig. 4A, black line; $\left.{ }^{*} \mathrm{p}<0.05\right)$. At the end of the study, tumors from the control group (no shRNA, $611.60 \mathrm{~mm}^{3}$ ) were significantly larger than the tumors from the knockdown group (OTX2 shRNA, $32.83 \mathrm{~mm}^{3}$; " $\mathrm{p}<0.05$ ). On average, tumors in the control group had a doubling time of 7 days, while tumors in the knockdown group had a doubling time of 15 days. To illustrate that the difference in tumor growth was mediated through inhibition of OTX2, IHC on tumors showed robust OTX2 expression in controls (80\% of cells with an intensity of 2+), while knockdown tumors showed less OTX2 expression (20\% of the cells with an intensity of 1+) (Fig. 4B).

OTX2 inhibition decreases MYC and CRX expression, but increases $p R B$ expression. To explore possible downstream effects of $O T X 2$ in retinoblastoma, we examined the effects of $O T X 2$ inhibition on the expression of genes previously suggested as OTX2 targets [MYC (11), CRX (22)], or implicated in majority of these tumors [phosphorylated RB (23)]. OTX2-specific siRNA knockdown in retinoblastoma resulted in the decreased expression of MYC and CRX but the increased expression of pRB by immunoblotting (Fig. 5A). Densitometry of the immunoblotting revealed that after OTX2 inhibition, the expression of MYC and CRX were decreased by $30 \%$ while pRB expression was increased by $40 \%$ (Fig. 5B).

\section{Discussion}

Current therapy for retinoblastoma, a malignant intraocular tumor in children, is non-specific and has many consequences. Because enucleation causes irreversible vision loss and because radiation therapy can result in the development of secondary malignancies in this young patient population, there is great interest in developing alternative chemotherapeutic agents as the primary form of treatment (24-27). A better understanding of specific genetic alterations in retinoblastoma could offer insight to potential targets for the treatment of this cancer. We recently identified $O T X 2$ as an oncogene for medulloblastoma, another common childhood malignant tumor $(10,11)$. OTX2 is a transcription factor that is crucial to the patterning of brain development and early brain morphogenesis, facilitates cell fate in the maturing retina $(14,15)$ and plays a role in tumorigenesis if aberrantly expressed later in life (12).

This study shows that the oncogene OTX2, previously demonstrated in medulloblastoma, may also play an important oncogenic role in retinoblastoma and can be targeted. These tumors have distinct characteristics and are typically not grouped together; yet, they do share some histological and genetic features. This study and others have now confirmed OTX2 as being frequently overexpressed in retinoblastoma primary tumors and cell lines, as well as alteration of related signaling networks (Fig. 6). In a study of 21 hereditary and non-hereditary retinoblastoma patients, Mol et al (28) found OTX2 gain and MYCN gain in non-hereditary tumors. Glubrecht et al (29) saw widespread overexpression of OTX2 and CRX in retinoblastoma tumors and cell lines and suggested a link between these and the retinoblastoma cell of origin. We further these observations by showing that OTX2 can be specifically targeted to reduce tumorigenesis in vitro and in vivo. Further experiments are necessary to clarify how abnormal OTX2 expression may result in the dysregulation of the developmental programs in retinal progenitor cells and cause neoplastic transformation.

It is of great interest that aberrant OTX2 expression affects other pathways implicated in retinoblastoma tumorigenesis (Fig. 6), suggesting possible mechanisms for initiating or maintaining tumorigenesis and a possible common therapeutic target. $C R X$ encodes for a transcription factor that is crucial to the differentiation of photoreceptor cells (30). A recent study by Omori et al (22) showed that OTX2 conditional knockout mice exhibited strong downregulation of CRX in the retina and here we show that CRX expression is decreased by specific OTX2 inhibition in retinoblastoma cells. OTX2 can actually directly regulate $C R X$ expression by binding to the cis-regulatory elements in its promoter region (20). MYC is an oncogene that promotes cell proliferation in many cancers and 

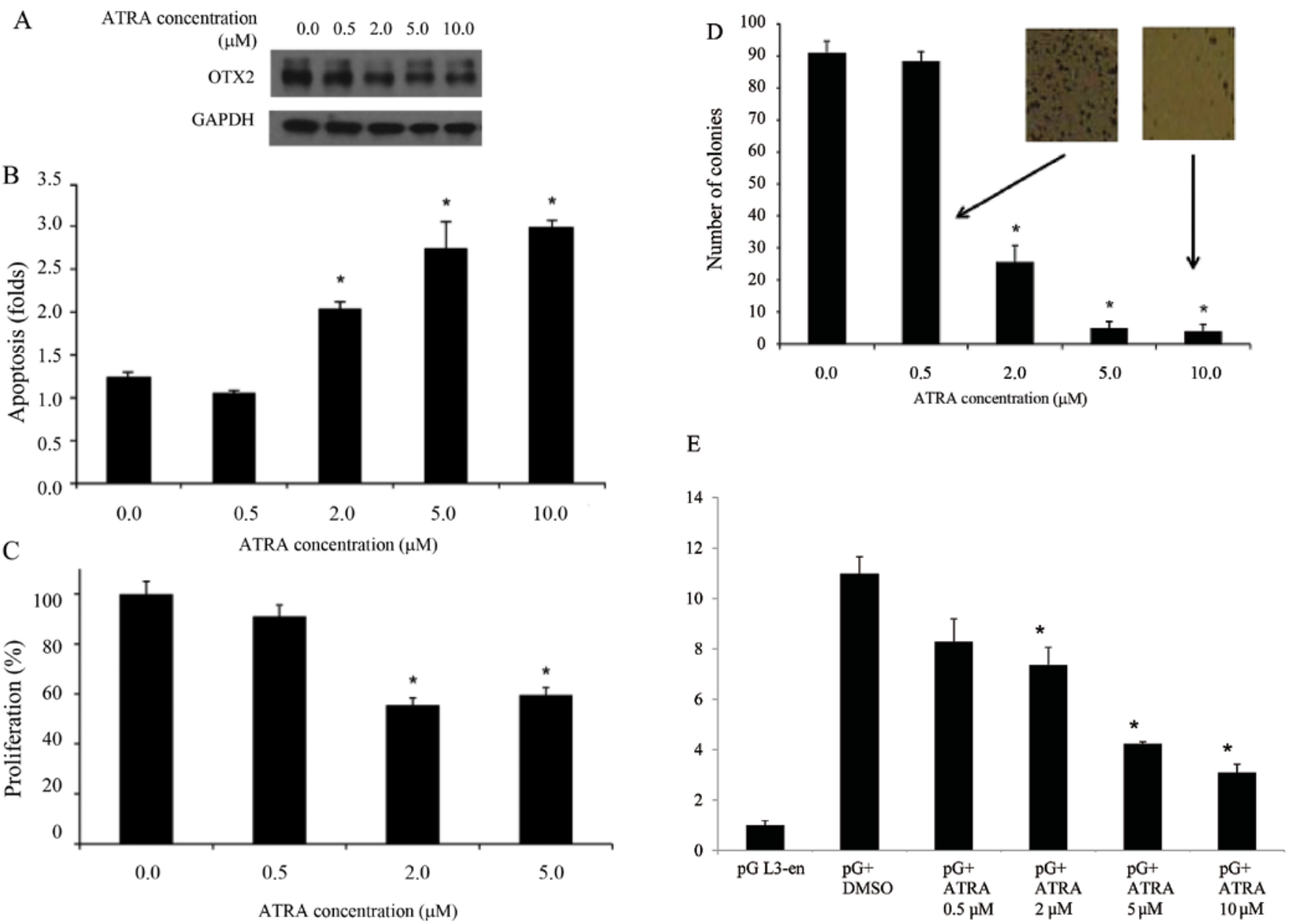

Figure 3. Pharmacologic inhibition of OTX2 with ATRA causes increased apoptosis and decreased proliferation and colony formation in retinoblastoma cell lines. (A) Representative immunoblotting shows successful OTX2 knockdown in Y79 after exposure to ATRA for $72 \mathrm{~h}$ in a dose-dependent manner. GAPDH served as housekeeping protein to demonstrate equal protein amounts. (B) ATRA induces retinoblastoma cell apoptosis. Y79 was treated with the indicated dose of ATRA $(0,0.5,2,5$ and $10 \mu \mathrm{M})$ for $72 \mathrm{~h}$ and apoptosis was measured. (C) OTX2 gene knockdown by ATRA decreases cell proliferation. Y79 was treated with the indicated dose of ATRA $(0,0.5,2$ and $5 \mu \mathrm{M})$ for $72 \mathrm{~h}$. The cells were allowed to grow for 7 days and the number of viable cells was determined by the MTT assay. (D) ATRA abrogated growth of retinoblastoma cells in soft agar. Y79 was incubated with the indicated dose of ATRA ( $0,0.5,2,5$ and $10 \mu \mathrm{M})$ for $72 \mathrm{~h}$ and plated at $5 \times 10^{3}$ in agar. After 2 weeks, the number of colonies was counted. Similar results were observed in WERI for studies in (A-D). (E) Luciferase activity from the OTX2 promoter construct decreased dose dependently with increasing amounts of ATRA. This was performed in HEK293 cells without endogenous OTX2. Asterisks (*) indicate significant differences when comparing each siRNA to the control ("p<0.05).
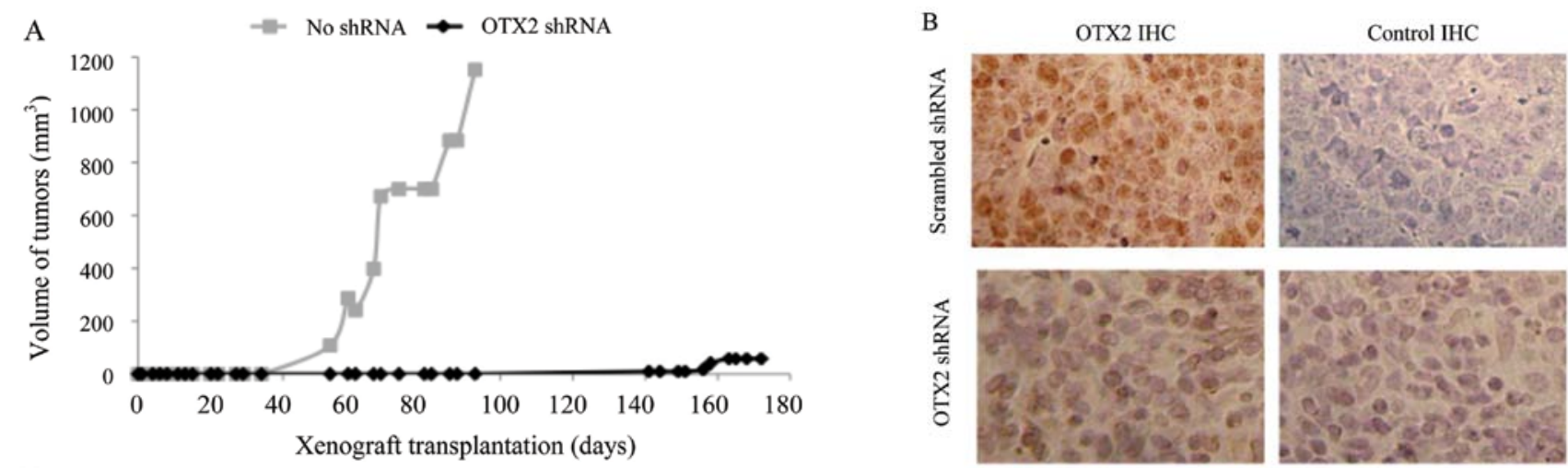

Figure 4. In vivo inhibition of OTX2 in retinoblastoma xenografts reduces tumor growth and tumor size significantly. (A) OTX2-specific shRNA inhibited tumor growth and tumor size. Mice that were injected with cells infected with OTX2-specific shRNA (OTX2 shRNA treatment group; black line, average data from 2 mice injected with WERI that was separately infected with viral vectors containing OTX2 shRNA\#1 and OTX2 shRNA\#2; both mice were given doxycycline) developed tumors that were significantly smaller at a significantly later time when compared with mice that were injected with uninfected cells (no shRNA control group; gray line, average data from 3 mice injected with uninfected Y79 and 1 mouse injected with uninfected WERI; all 4 mice were given doxycycline) $(\mathrm{p}<0.05)$. (B) Representative IHC staining for OTX2 and control (no primary antibody) in paraffin-embedded mouse tumors $(\mathrm{x} 40)$. IHC shows robust OTX2 staining when OTX2 is not knocked down (top row) and weak OTX2 staining when OTX2 is knocked down (bottom row). Top row, tumor was obtained from the mouse injected with WERI that was infected with viral vector containing scrambled shRNA; bottom row, tumor was obtained from the mouse infected with WERI that was infected with viral vector containing OTX2 shRNA\#2; both mice were given doxycycline. 


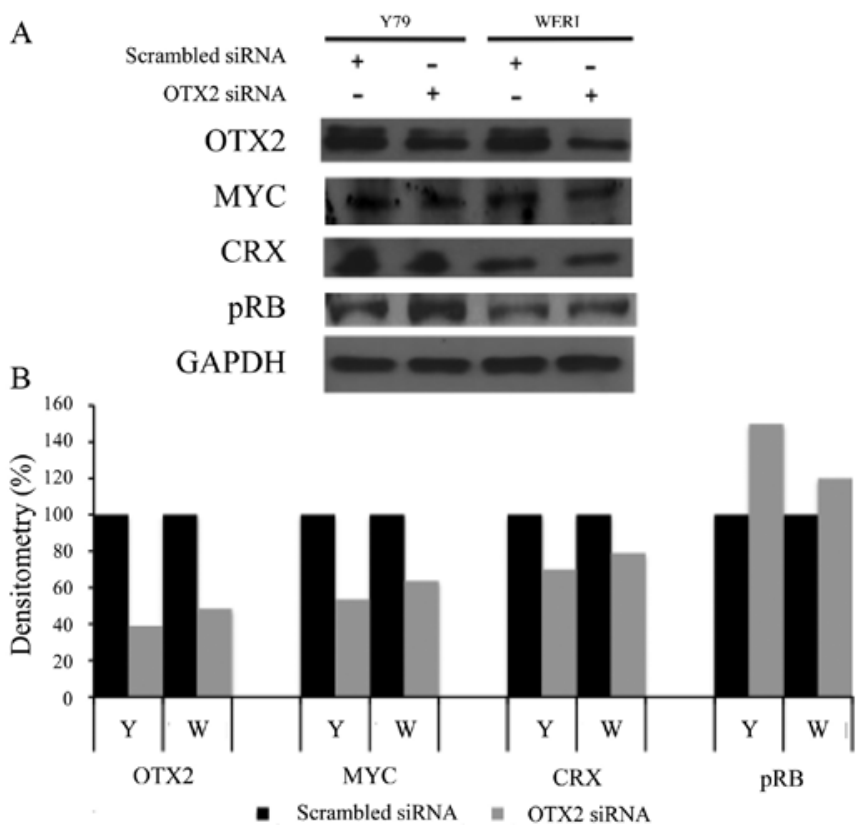

Figure 5. OTX2 inhibition reduces MYC and CRX expression, but increases pRB expression. (A) Representative immunoblotting shows that when Y79 and WERI were treated with OTX2 siRNA, OTX2, MYC and CRX expression decreased but phosphorylated RB expression increased. GAPDH served as housekeeping protein to demonstrate equal protein amounts. (B) Densitometry of immunoblotting results reveal that when OTX2 expression was inhibited by siRNA, MYC and CRX expression also decreased while pRB expression increased, done in triplicate with error bars. is a known downstream target of oncogenic OTX2 $(11,31)$. Our previous study showed that OTX2 also directly upregulates the expression of $M Y C$ in medulloblastoma via cis-regulatory elements in the MYC promoter region (11). $R B$ is a well-known tumor suppressor gene for retinoblastoma and other cancers that inhibits cell cycle progression and suppresses cell growth (32). Bunt et al (23) showed that OTX2 expression in medulloblastoma cells decreased the levels of phosphorylated RB (pRB). Consistent with this result, our results showed that OTX2 inhibition increased pRB levels in retinoblastoma. Previous studies by others support the possibility that OTX2 regulates the expression of all three genes $(11,22,23)$ and it will be interesting to further clarify the role of OTX2 in these signaling networks. As seen here, directly targeting OTX2 or these related factors may be a viable option with siRNA-like strategies. Alternatively, pharmacologic agents that can drive terminal differentiation of tumor cells may have a beneficial impact on curtailing uncontrolled growth of tumors. For example, retinoic acid can drive photoreceptor cell differentiation (33). It is conceivable that retinoid agents may combat tumorigenesis via multiple mechanisms, e.g., by specifically inhibiting oncogenic factors like OTX2 and at the same time by promoting the expression of differentiating factors in retinoblastoma. In our study, $2 \mu \mathrm{M}$ ATRA consistently decreased proliferation and colony formation, while increasing cell death. Furthermore, ATRA specifically repressed OTX2 expression at its promoter. Although ATRA and other retinoids may affect multiple molecular pathways, the connection between OTX2

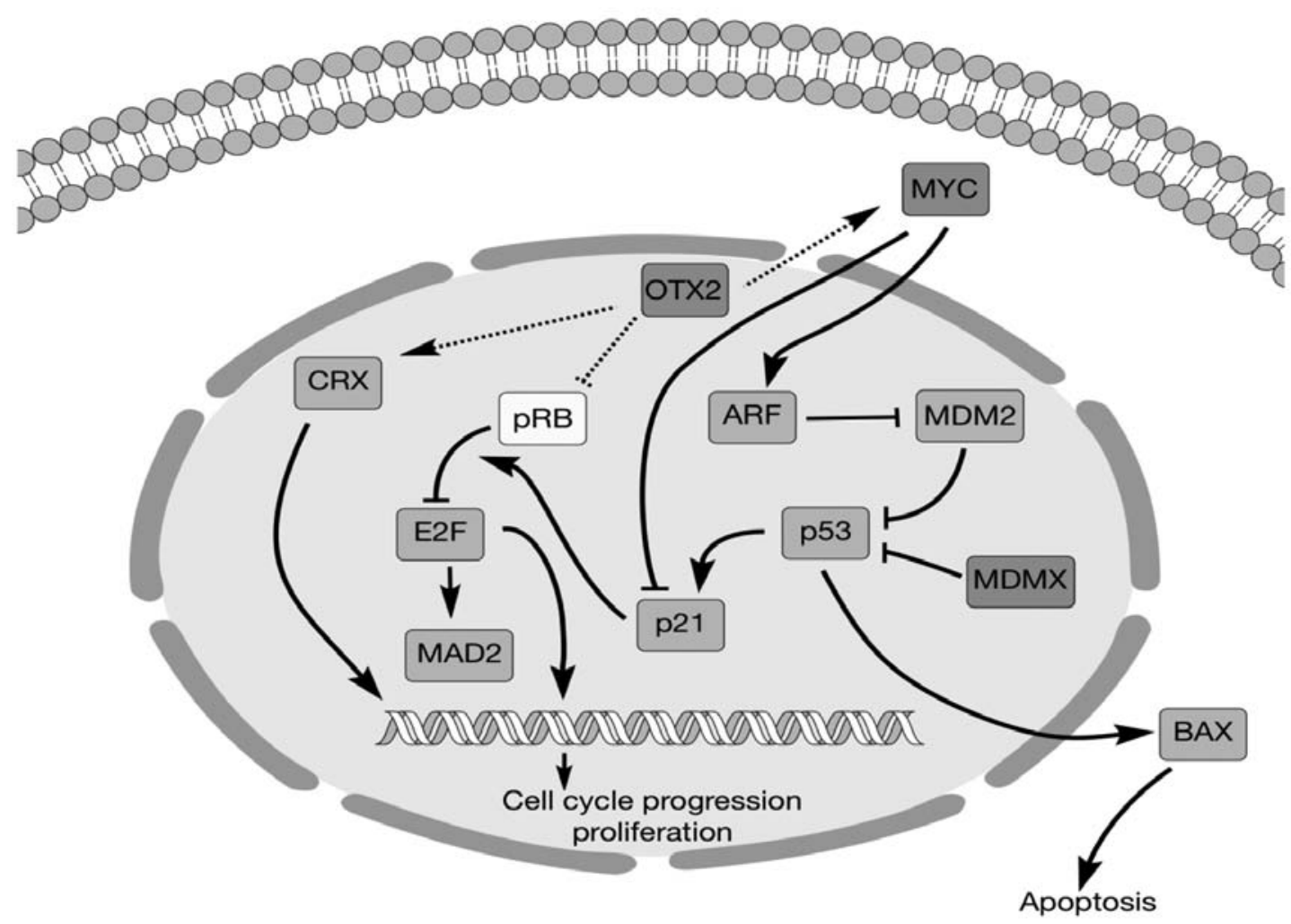

Figure 6. OTX2 may serve as a common transcription factor interlinking multiple retinoblastoma signaling pathways. Representative retinoblastoma cell showing some common alterations in genetic pathways. Main pathways to consider include the RB pathway, the p53 pathway and the MYC pathway. Solid lines represent known interactions between molecules, dotted lines represent possible interactions between pathways based on the present study. Lighter circles represent molecules with less expression in retinoblastoma, darker circles represent molecules with more expression in retinoblastoma. 
repression and growth inhibition effect of ATRA suggests that OTX2 expressing tumors may be amenable to therapy with minimal doses of retinoids that are less toxic. ATRA is approved clinically for the treatment of acute promyelocytic leukemia, a disease in which another nuclear receptor is a predominant determinant of pathogenesis.

Our studies of OTX2, in conjunction with the studies of others, lay the conceptual framework for clinical trials of retinoids and $O T X 2$-specific agents in the treatment of this challenging pediatric tumor. The opportunity to develop new targeted therapeutic agents against retinoblastoma is of great interest. Current treatments of enucleation followed by non-specific adjuvant therapies may cure the tumor, but at grave costs. Loss of vision is universal and the long-term toxicities of these adjuvant therapies on this young patient population can be devastating. More specific agents may be less toxic.

\section{References}

1. Wilson WG: Retinoblastoma. Pediatr Rev 28: 37-38, 2007.

2. Eldebawy E, Parker W, Abdel Rahman W and Freeman CR: Dosimetric study of current treatment options for radiotherapy in retinoblastoma. Int J Radiat Oncol Biol Phys 82: e501-e505, 2012.

3. Zhang J, Benavente CA, McEvoy J, Flores-Otero J, Ding L, Chen X, Ulyanov A, Wu G, Wilson M, Wang J, et al: A novel retinoblastoma therapy from genomic and epigenetic analyses. Nature 481: 329-334, 2012.

4. Devesa SS: The incidence of retinoblastoma. Am J Ophthalmol 80: 263-265, 1975.

5. Bhagia P, Colanta AB, Abramson DH, Carlson DL, Kleinerman RA Kraus D and Dunkel IJ: Sinonasal adenocarcinoma: a rare second malignancy in long term retinoblastoma survivors. Pediatr Blood Cancer 57: 693-695, 2011.

6. Draf C, Schaberg MR, Anand VK, Nyquist G and Hoda S: Radiation induced malignancy in retinoblastoma: new pathology in a case report. Laryngoscope 120 (Suppl 4): S238, 2010.

7. Lohmann D: Retinoblastoma. Adv Exp Med Biol 685: 220-227, 2010.

8. Abramson DH, Melson MR, Dunkel IJ and Frank CM: Third (fourth and fifth) nonocular tumors in survivors of retinoblastoma. Ophthalmology 108: 1868-1876, 2001.

9. Eng C, Li FP, Abramson DH, Ellsworth RM, Wong FL, Goldman MB, Seddon J, Tarbell N and Boice JD Jr: Mortality from second tumors among long-term survivors of retinoblastoma. J Natl Cancer Inst 85: 1121-1128, 1993.

10. Di C, Liao S, Adamson DC, Parrett TJ, Broderick DK, Shi Q, Lengauer C, Cummins JM, Velculescu VE, Fults DW, et al: Identification of OTX2 as a medulloblastoma oncogene whose product can be targeted by all-trans retinoic acid. Cancer Res 65 919-924, 2005

11. Adamson DC, Shi Q, Wortham M,Northcott PA, Di C, Duncan CG, Li J, McLendon RE, Bigner DD, Taylor MD, et al: OTX2 is critical for the maintenance and progression of Shh-independent medulloblastomas. Cancer Res 70: 181-191, 2010.

12. Mattox A, Li J, Di C and Adamson DC: Medulloblastoma: role of OTX2 transcription factors. In: Tumors of the Central Nervous System. Vol. 8. Hayat MA (ed). Springer Science, 2012.

13. Martinez-Morales JR, Dolez V, Rodrigo I,Zaccarini R, Leconte L, Bovolenta P and Saule S: OTX2 activates the molecular network underlying retina pigment epithelium differentiation. J Biol Chem 278: 21721-21731, 2003.
14. Nishida A, Furukawa A, Koike C, Tano Y, Aizawa S, Matsuo I and Furukawa T: Otx2 homeobox gene controls retinal photoreceptor cell fate and pineal gland development. Nat Neurosci 6: 1255-1263, 2003.

15. Sato S, Inoue T, Terada K, Matsuo I, Aizawa S, Tano Y, Fujikado T and Furukawa T: Dkk3-Cre BAC transgenic mouse line: a tool for highly efficient gene deletion in retinal progenitor cells. Genesis 45: 502-507, 2007.

16. Elias WJ, Lopes MBS, Golden WL, Jane JA and GonzalezFernandez F: Trilateral retinoblastoma variant indicative of the relevance of the retinoblastoma tumor-suppressor pathway to medulloblastomas in humans J Neurosurg 95: 871-878, 2001.

17. Jurkiewicz E, Pakuła-Kościesza I, Rutynowska $\mathrm{O}$ and Nowak $\mathrm{K}$ : Trilateral retinoblastoma: an institutional experience and review of the literature. Child's Nervous System 26: 129-132, 2010.

18. Mouratova T: Trilateral retinoblastoma: a literature review, 1971-2004. Bull Soc Belge Ophtalmol 297: 25-35, 2005.

19. Jaffey PB, To GT, Xu HJ, Hu SX, Benedict WF, Donoso LA, and Campbell GA: Retinoblastoma-like phenotype expressed in medulloblastomas. J Neuropathol Exp Neurol 54: 664-672, 1995

20. Santagata S, Maire CL, Idbaih A, Geffers L, Correll M, Holton K, Quackenbush J and Ligon KL: CRX is a diagnostic marker of retinal and pineal lineage tumors. PLoS One 4: e7932, 2009.

21. Stephan H,Zakrzewski JL, Bölöni R, Grasemann C, Lohmann DR and Eggert A: Neurotrophin receptor expression in human primary retinoblastomas and retinoblastoma cell lines. Pediatr Blood Cancer 50: 218-222, 2008.

22. Omori Y, Katoh K, Sato S, Muranishi Y, Chaya T, Onishi A, Minami T, Fujikado T and Furukawa T: Analysis of transcriptional regulatory pathways of photoreceptor genes by expression profiling of the Otx2-deficient retina. PLoS One 6: e19685, 2011.

23. Bunt J, De Haas TG, Hasselt NE, Zwijnenburg DA, Koster J, Versteeg R and Kool M: Regulation of cell cycle genes and induction of senescence by overexpression of OTX2 in medulloblastoma cell lines. Mol Cancer Res 8: 1344-1357, 2010.

24. Wong FL,Boice JDJr, Abramson DH, Tarone RE,Kleinerman RA, Stovall M, Goldman MB, Seddon JM, Tarbell N, Fraumeni JF Jr, et al: Cancer incidence after retinoblastoma. Radiation dose and sarcoma risk. JAMA 278: 1262-1267, 1997.

25. Mohney BG, Robertson DM, Schomberg PJ and Hodge DO: Second nonocular tumors in survivors of heritable retinoblastoma and prior radiation therapy. Am J Ophthalmol 126: 269-277, 1998.

26. Smith LM, Donaldson SS, Egbert PR, Link MP and Bagshaw MA: Aggressive management of second primary tumors in survivors of hereditary retinoblastoma. Int J Radiat Oncol Biol Phys 17: 499-505, 1989.

27. Shields CL and Shields JA: Retinoblastoma management: advances in enucleation, intravenous chemoreduction, and intra-arterial chemotherapy. Curr Opin Ophthalmol 21: 203-212, 2010.

28. Mol BM, Massink MP, van der Hout AH, Dommering CJ, Zaman JM, Bosscha MI, Kors WA, Meijers-Heijboer HE, Kaspers GJ, Riele Ht, et al: High resolution SNP array profiling identifies variability in retinoblastoma genome stability. Genes Chromosomes Cancer 53: 1-14, 2014.

29. Glubrecht DD, Kim JH, Russell L, Bamforth JS and Godbout R: Differential CRX and OTX2 expression in human retina and retinoblastoma. J Neurochem 111: 250-263, 2009.

30. Furukawa T, Morrow EM and Cepko CL: Crx, a novel otx-like homeobox gene, shows photoreceptor-specific expression and regulates photoreceptor differentiation. Cell 91: 531-541, 1997.

31. Nilsson JA and Cleveland JL: Myc pathways provoking cell suicide and cancer. Oncogene 22: 9007-9021, 2003.

32. Wiman K: The retinoblastoma gene: role in cell cycle control and cell differentiation. FASEB J 7: 841-845, 1993.

33. Khanna H, Akimoto M, Siffroi-Fernandez S, Friedman JS, Hicks D and Swaroop A: Retinoic acid regulates the expression of photoreceptor transcription factor NRL. J Biol Chem 281: 27327-27334, 2006. 